\title{
La place des messagers et des ambassadeurs dans la diplomatie princière à la fin du Moyen Age
}

Jean-Marie Moeglin

\section{OpenEdition}

Édition électronique

URL : http://journals.openedition.org/edl/249

DOI : $10.4000 /$ edl. 249

ISSN : 2296-5084

Éditeur

Université de Lausanne

\section{Édition imprimée}

Date de publication : 15 septembre 2010

Pagination : 11-36

ISBN : 978-2-940331-23-9

ISSN : 0014-2026

\section{Référence électronique}

Jean-Marie Moeglin, «La place des messagers et des ambassadeurs dans la diplomatie princière à la fin du Moyen Age », Études de lettres [En ligne], 3 | 2010, mis en ligne le 15 septembre 2013, consulté le 18 décembre 2020. URL : http://journals.openedition.org/edl/249; DOI : https://doi.org/10.4000/edl. 249 


\section{LA PLACE DES MESSAGERS ET DES AMBASSADEURS DANS LA DIPLOMATIE PRINCIÈRE À LA FIN DU MOYEN AGE}

Les légats et ambassadeurs dans la diplomatie des rois, princes et autres autorités supérieures ont fait l'objet d'un petit nombre de travaux anciens ${ }^{1}$ ou moins anciens ${ }^{2}$, et plus récemment d'une série de recherches ${ }^{3}$ qui permettent désormais d'évoquer avec quelque précision

I. V. Menzel, Deutsches Gesandschaftswesen im Mittelalter; R. de Maulde de La Clavière, La diplomatie au temps de Machiavel.

2. F. Ernst, "Über Gesandschaftswesen und Diplomatie an der Wende vom Mittelalter zur Neuzeit»; G. Mattingly, Renaissance Diplomacy; D. Queller, "Thirteenth-Century Diplomatic Envoys"; Id., Early venetian Legislation on Ambassadors; Id., The office of Ambassador in the Middle Ages; W. Höflechner, Beiträge zur Geschichte der Diplomatie und Gesandschaftswesen unter Maximilian I.; Id., "Anmerkungen zu Diplomatie und Gesandschaftswesen des 15. Jahrhunderts»; Id., «Die Entwicklung österreichischer Diplomatie im Mittelalter und die Aussenpolitik Maximilians I.»; P. Chaplais, English Medieval Diplomatical Practice (Part I Documents and Intepretation); Id., English Diplomatic Practice in the Middle Ages.

3. Cf. notamment J. Petersohn, Ein Diplomat des Quattrocento.; Id., Kaiserlicher Gesandter und Kurienbischof; I. Le Bis, "Pratique de la diplomatie.»; R. Fubini, "Classe dirigente e esercizio dalla diplomazia nella Firenze quattrocentesca»; Id., Quattrocento fiorentino. Politica diplomazia cultura; P. Savy, «Les ambassadeurs milanais à la cour de Charles le Téméraire»; Th. Behrmann, Herrscher und Hansestädte; Id., "Hansische Gesandte an Herrscherhöfen»; Id., "Vor Stralsund (1370) und Vordingborg (1425)»; Ch. de Borchgrave, "Diplomates et diplomaties sous le duc de Bourgogne Jean sans Peur"; Ch. Lutter, Politische Kommunikation an der Wende vom Mittelalter zur Neuzeit; J. M. Currin, "„Pro expensis Ambassatorum “»; A. Reitemeier, Aussenpolitik im Spätmittelalter; M. Kintzinger, Westbindungen im spätmittelalterlichen Europa; P. Ehm, Burgund und das Reich; F. Autrand, "Ambassade»; R. A. Schwinges und K. Wriedt (Hrsg.), Gesandschafts- und Botenwesen im spätmittelalterlichen Europa; M. Prietzel, "Reden als Waffen der Diplomatie»; E. Pibiri, "A la recherche d'une épouse"; Ead., "En ambassarie devers le roi des Romains"; Ead., "Etre reçu à cour: 
la question de leur place et de leur rôle à la frontière du Moyen Age et de l'époque moderne.

L'importance de cette période dans l'histoire de la diplomatie a certes été soulignée depuis longtemps, mais elle l'a été dans une perspective que des travaux récents conduisent à relativiser fortement voire à remettre en question; il s'agit de l'idée selon laquelle cette période aurait vu l'émergence de l'ambassadeur résident, représentant d'un Etat auprès d'un autre Etat, mettant fin au système des ambassades ad hoc d'un prince vers un autre prince, et inaugurant ainsi l'avènement, à proprement parler, de la diplomatie professionnelle moderne. Une telle conception ne peut effectivement plus être retenue telle quelle, d'abord parce que les ambassadeurs résidents ont mis beaucoup de temps à se concevoir comme tels et qu'il ne s'agit en aucune façon d'une apparition consciemment planifiée, ensuite parce que les ambassades ad hoc restent tout au long de l'époque moderne, et encore maintenant au demeurant, un pilier essentiel de la diplomatie des Etats ${ }^{4}$.

Pour autant, il se pourrait malgré tout que les $\mathrm{XV}^{\mathrm{e}}-\mathrm{XVI} \mathrm{I}^{\mathrm{e}}$ siècles représentent un moment essentiel dans l'affirmation d'une diplomatie fondée sur les ambassadeurs, l'apparition d'ambassadeurs quelque peu permanents ne représentant qu'un aspect très partiel de ce processus.

Cela me paraît en effet la conséquence d'une transformation que l'on a mise en évidence sans en mesurer peut-être toutes les conséquences; il s'agit de la disparition progressive, du XV ${ }^{\mathrm{e}}$ au XVI $\mathrm{XV}^{\mathrm{e}}$ siècle, des rencontres de princes. Or ces rencontres étaient restées tout au long du Moyen Age le cœur des relations entre princes et états. Un exemple tout à fait banal est celui du traité conclu le 13 juillet 1288, sous la médiation du cardinal Jean Cholet, entre les rois Philippe le Bel et Sanche IV de Castille pour mettre fin au long conflit entre les rois des deux pays dû aux "enfants de la Cerda»; le document détaille les stipulations de l'accord

l'accueil des ambassadeurs étrangers par les ducs Amédée VIII et Louis de Savoie»; Ead., En voyage pour Monseigneur; K. Plöger, England and the Avignon Popes; P. Gilli, "La fonction d'ambassadeur dans les traités juridiques italiens du XVe siècle»; Id., "De l'importance d'être hors norme»; S. Péquignot, "Les instructions aux ambassadeurs des rois d'Aragon (XII -XVe siècles)»; Id., Au nom du roi; A.-B. Spitzbarth, Ambassades et ambassadeurs de Philippe le Bon, troisième duc Valois de Bourgogne (1419-1467);

C. Zey und C. Märtl (Hrsg.), Aus der Frühzeit europäischer Diplomatie.

4. Pour la critique de la conception ancienne, cf. en dernier lieu S. Péquignot, «Les diplomaties occidentales et le mouvement du monde», en part. p. 712-713. 
avant de préciser «et pro istis omnibus et singulis firmandis et plenius adimplendis, prefati Francie et Castelle reges debent se invicem videre in aliquo certo loco de communi eorum voluntate ac beneplacito assignando " 5 . Toutes les clauses de l'arrangement étaient donc prêtes mais le traité ne pouvait entrer en vigueur avant d'avoir été scellé par les engagements personnels des deux souverains l'un envers l'autre et plus généralement par la restauration entre eux d'une relation d'amour mutuel. Cet exemple n'a rien d'exceptionnel; on pourrait par exemple écrire l'histoire de la Guerre dite de Cent ans comme celle d'une rencontre de rois sans cesse reportée à la recherche de la "paix finale».

Dans la première moitié du XVI $\mathrm{X}^{\mathrm{e}}$ siècle cependant, il est clair que ces rencontres, sous leur forme médiévale classique, sont en train de prendre fin; si l'on prend le cas des rois de France ${ }^{6}$, on ne peut plus citer que quelques épisodes : la fameuse rencontre du drap d'or en 1520 entre François I $^{\text {er }}$ et Henri VIII; une nouvelle rencontre entre ces deux souverains en 1532 à Marquise; deux rencontres entre le roi de France et le pape, en 1515 d'abord à Bologne puis en 1533 à Marseille; une rencontre entre François I ${ }^{\text {er }}$ et Charles Quint en 1538 à Nice. Par la suite, les rencontres de princes organisées par le roi de France changèrent de nature: il s'agissait désormais d'accueillir dans l'honneur des rois et des reines chassés de leur royaume, des filles de France venant se réfugier dans leur pays natal, des princes protestants dont on recherchait l'alliance.

Ce déclin fait bien sûr songer au mot fameux et souvent cité de Commynes sur ces "grandes folies" qu'étaient les rencontres de rois. Elles étaient néanmoins longtemps restées l'étape fondamentale de la relation diplomatique, indispensables pour que des engagements fermes soient pris grâce au lien d'amour qu'elles seules permettaient de conclure et sans lequel aucun traité ne pouvait être considéré comme véritablement ratifié. Mais désormais leur organisation devenait bien trop difficile; elle se heurtait au processus général de sédentarisation, d'étatisation et de bureaucratisation dans les royaumes et principautés.

Il n'est donc pas sans intérêt de se demander si cet effacement progressif des rencontres des princes ne pourrait pas avoir contribué à projeter au premier plan de la scène diplomatique les ambassadeurs et légats

5. G. Daumet, Mémoires sur les relations de la France et de la Castille, p. 197.

6. L. Bély, La société des princes, XVI ${ }^{e}-X V I I I^{e}$ siècle, en part. p. 387-395. 
des princes, avoir poussé à les doter d'un véritable statut tandis que se précisaient une hiérarchie et une différenciation fonctionnelle entre les divers types de représentants du prince actifs dans les relations diplomatiques.

L'ambassadeur était en effet resté tout au long du Moyen Age une sorte de personnage schizophrène. Il était l'homme qui aurait dû se transformer en un autre, devenir le double de son prince, littéralement le re-présenter, s'effacer totalement derrière lui jusqu'à en reprendre la personnalité; mais en même temps, il ne parvenait pas à faire oublier qu'il n'était précisément que le pâle substitut de son maître absent, incapable de se prévaloir de l'autorité attachée à la personne propre de ce dernier.

La question que l'on voudrait poser ici est de savoir dans quelle mesure l'ambassadeur s'est trouvé en mesure, à la fin du Moyen Age et au début de l'époque moderne, à la faveur de l'effacement des rencontres de princes, de surmonter ces obstacles, d'acquérir un statut qui lui permette désormais de rencontrer le prince étranger, de parler et de s'engager à la place de son mandataire, exactement comme s'il avait été celui-ci.

\section{L'ambassadeur substitut de son maître}

L'ambassadeur n'était donc qu'un substitut à la présence réelle de son prince. Mais il va de soi que les princes ne pouvaient être partout, que leurs rencontres étaient complexes à organiser, toujours risquées; elles ne pouvaient par conséquent qu'être relativement peu fréquentes; par ailleurs, la complexité des affaires à traiter faisait qu'elles ne pouvaient l'être entièrement en une unique rencontre qu'il fallait de toute façon organiser à l'avance. L'ambassadeur est donc celui qui, en partie, prépare la future rencontre des princes, en partie représente le prince et accomplit ainsi une part du travail que celui-ci aurait mené à bien s'il avait été présent; un recours par défaut par conséquent, mais qui se révèle malgré tout essentiel.

Cela définit en tout cas le cadre d'ensemble de l'activité des légats et des ambassadeurs, celui du substitutif et du provisoire, celui du ponctuel, bref celui des légations ad hoc effectuées par des gens qui ne sont ni des officiers, ni des magistrats et qui n'ont pas d'autre statut que celui de représenter la personne de leur maître qui ne peut ou ne veut lui-même venir rencontrer son partenaire étranger. 
Il leur incombe par conséquent en premier lieu d'obtenir accès à la personne de l'autre prince. Cela, en fait, se révèle bien souvent extrêmement difficile, d'abord pour des raisons techniques, du fait de l'itinérance persistante des cours. Les envoyés vénitiens à la cour de Maximilien, dans les dispacci qu'ils envoient au sénat, se plaignent ainsi souvent d'être à la poursuite d'un souverain parti à la chasse (seuls ses proches conseillers savent où il se trouve), ou bien occupé à régler des affaires plus importantes et donc inaccessible. Le 18 août 1496, Francesco Foscari, ambassadeur de Venise auprès de Maximilien, se désole de n’avoir pas connu un seul moment de repos pendant trois mois (" non ho mai saputo che sia riposo"), pour ne pas parler de tout ce qu'il a dû endurer physiquement ("le fatiche e i sinistri corporali»), tandis que dans la relation de son ambassade des années 1501 et 1502 chez Maximilien, Zaccaria Contarini se plaint de n'avoir pas pu en 17 mois séjourner plus de 5 jours d'affilé dans un même endroit, ceci pendant 17 mois! ${ }^{7}$

Mais ce n'était pas le seul obstacle que l'ambassadeur devait affronter pour pouvoir rencontrer le prince: un envoyé pouvait avoir à acheter la bienveillance des conseillers du souverain auquel il rendait visite, rien que pour accéder au destinataire de son ambassade. Bonifatius de Cuppis, envoyé du comte de Mantoue chez Venceslas, dut payer 100000 ducats à différents proches du souverain simplement pour avoir le droit de présenter sa demande. Contre la promesse de 20000 ducats il obtint finalement au moins l'assurance que les documents qu'il demandait lui seraient établis ${ }^{8}$. Un serviteur du comte de Saint-Pol se plaignait en 1468 qu'on ne pouvait obtenir une audience de Charles qu'en passant par son favori, le "mignone de camera", en l'occurrence, semblet-il, Guillaume Bische 9 . Le 23 juin 1497, l'ambassadeur vénitien Giorgio Pisani chez Maximilien recevait comme instructions secrètes d'acheter la bienveillance des personnes qui comptaient à la cour de l'empereur; il en allait de fait d'une affaire importante puisqu'il s'agissait de l'obtention du comté de Görz; il était autorisé à donner jusqu'à 2000 ducats en cadeau aux conseillers et secrétaires de la cour impériale (un ambassadeur vénitien recevait entre 120 et 150 ducats par mois tandis que le salaire d'un

7. Ch. Lutter, Politische Kommunikation an der Wende vom Mittelalter zur Neuzeit, passim.

8. A. Reitemeier, Aussenpolitik im Spätmittelalter, p. 457.

9. P. Ehm, Burgund und das Reich, p. 268. 
secrétaire de la chancellerie du doge se situait annuellement entre 30 et 60 ducats) ${ }^{10}$. Les ambassadeurs permanents ou quasi permanents s'efforçaient de se créer un réseau d'amitiés à la cour qui leur facilitait l'accès au prince; ainsi l'ambassadeur du seigneur de Milan à la cour de Charles le Téméraire, Panigarola, se trouve en difficulté lorsque meurt son "contact" privilégié, le médecin italien Matteo di Troya; il choisit alors de s'attacher les services d'un groupe de quatre personnes dont les compétences se complétaient: le médecin portugais Lupo, le secrétaire Jean Coulon, le seigneur de Contay Louis le Jeune et le portier Henri d'Anvers ${ }^{11}$.

La difficulté de l'accès au souverain restait bien à la fin du Moyen Age une des grandes limites de l'efficacité de la diplomatie des ambassades ad hoc; l'émergence des ambassadeurs permanents, si l'on peut considérer comme tels les ambassadeurs vénitiens à la cour de Maximilien, n'avait pas apporté de véritable solution à ce problème. La capacité des ambassadeurs a franchir rapidement cet obstacle était en tout cas le premier test qu'ils devaient passer pour démontrer leur compétence ${ }^{12}$.

\section{Les objectifs d'une légation: la vaste palette de la "communication"}

Dans la fonction de "représentation» de son maître, une vaste palette de tâches concrètes attendait l'ambassadeur.

Il lui fallait d'abord assurer littéralement la représentation de son maître, en incarner la grandeur et la majesté, impressionner le destinataire; il s'agissait d'un rôle fondamental. Il influait d'abord sur le choix de certains membres de l'ambassade recrutés en raison de leur rang éminent; mais cette nécessité de représentation se traduisait aussi dans les cadeaux que tout ambassadeur devait apporter et qui illustraient l'importance de son maître en même temps qu'ils contribuaient à créer un lien d'amour entre les deux princes. Un jeu subtil pouvait ainsi se développer entre l'ambassadeur et la cour récipiendaire dans une sorte de concours

Io. Ch. Lutter, Politische Kommunikation an der Wende vom Mittelalter zur Neuzeit, p. 49.

II. P. Ehm, Burgund und das Reich, p. 268.

I2. Cf. par exemple M. Prietzel, «Reden als Waffen der Diplomatie», p. 77, à propos de l'ambassade bourguignonne à la fin novembre 1456 auprès de Charles VII dans le contexte très délicat de la fuite du futur Louis XI chez Philippe le Bon. 
de bonnes manières visant à s'impressionner mutuellement. Déjà une anecdote célèbre de Notker le Bègue avait illustré cet aspect; il rapporte comment la cour de Charlemagne lequel, comme tous ses successeurs, souffrait d'un complexe d'infériorité vis-à-vis des empereurs byzantins, avait joué un mauvais tour aux ambassadeurs de Constantinople; elle les avait fait comparaître successivement devant toute une série de dignitaires de la cour qu'ils prenaient à chaque fois pour l'empereur luimême, avant de leur donner le coup final en les introduisant devant un Charlemagne resplendissant d'or et de pierres précieuses ${ }^{13}$. Les questions de protocole jouent en tout état de cause un rôle important, voire décisif; selon son biographe Naldo Naldi, le Florentin Giannozzo Manetti, aurait provoqué un incident diplomatique grave lors de l'ambassade qu'il dirigeait en 1445 à Naples pour le mariage de Ferrant, fils du roi Alphonse; ayant constaté que les ambassadeurs génois avaient pris place à côté du roi, devant les Florentins, il aurait sur-le-champ quitté l'église et répondu au monarque qui lui demandait la raison de ce départ précipité, qu'il était indigne pour le représentant d'une grande république de passer après les envoyés d'un Etat subordonné ${ }^{14}$.

L'ambassadeur avait ensuite à traiter les negotia de son maître; il ne s'agissait au départ que d'être le porteur de la parole ou des secrets de son maître, ceux que son maître n'avait pu transmettre lui-même; le nuntius n'est étymologiquement pas autre chose que celui qui annonce de même que le terme allemand Botschafter signifie littéralement «celui qui transmet une nouvelle ou un ordre». Mais il va de soi que ces negotia allaient bien au-delà de la simple transmission de la parole du prince. Dans les années 1340 , le pape invitait le roi Philippe VI à entretenir comme les autres princes un représentant permanent à Avignon qui serait le "negotiorum suorum promotorem ${ }^{15}$. Edouard III qualifiait quant à lui les représentants qu'il envoyait de "nostros negotiorum gestores" ${ }^{16}$. Il en résulte une grande variété de messagers et ambassadeurs et une multiplicité de termes pour les désigner, des simples messagers ${ }^{17}$, appelés cursores

13. A. Mohr, Das Wissen über die anderen, p. 223 sqq.

I4. P. Gilli, «De l'importance d'être hors norme», p. 425.

I5. Cité par F. J. Felten, "Verhandlungen an der Kurie im frühen 14. Jahrhundert», p. 437 , n. 115 .

16. Foedera, ed. T. Rymer, passim.

17. Sur les messagers, tout particulièrement urbains, cf. les travaux récents ou en cours de publication de K. Hübner, "Nüwe mer us Lamparten» et P. Monnet, Recherches 
à la cour d'Angleterre, aux solempnes nuntii, sans que ces variations de désignation renvoient toujours à une différenciation parfaitement claire de leurs missions.

Le caractère de plus en plus technique des missions diplomatiques investies des negotia de leurs mandants s'accentuait et créait une pression en faveur du recrutement de spécialistes; une étude de la diplomatie hanséate montre qu'entre 1360 et 1460 , les règles du jeu se sont modifiées: en 1360, le roi Waldemar du Danemark «négociait» directement avec les représentants hanséates la concession de privilèges et cette négociation conduisait à la concession de la grâce et de la bienveillance royale au terme de tractations directes entre le souverain et les maires des villes. Un siècle plus tard, c'est une argumentation juridique qui domine les négociations et les délégations hanséates ne peuvent plus se déplacer sans se faire accompagner de juristes hautement qualifiés ${ }^{18}$. On constate dans ces conditions que le vieux face-à-face souverain/maires des villes de la Hanse est désormais relativisé par le recours à des juristes et à leur argumentation. Les débâcles diplomatiques des villes de la Hanse en 1434 et 1435 lors de négociations avec les Anglais furent expliquées par le comptoir de Bruges par le fait que les ambassadeurs auraient gravement souffert de l'absence d'un juriste ("groet gehebreck eens gheleerden mans of doctors") ${ }^{19}$; elles eurent en tout cas un effet salutaire: en 1436 les maires se firent épauler par le docteur en droit canon Franko Keddeken, chanoine de Saint-Donatien de Bruges, et le chancelier de

sur l'information, la communication et la représentation extérieure des villes allemandes à la fin du Moyen Age.

I8. Th. Behrmann, Herrscher und Hansestädte, p. 320 ; cf. aussi K. Wriedt, «Das gelehrte Personal in der Verwaltung und Diplomatie der Hansestädte».

19. Les envoyés de la Hanse (cf. A. Reitemeier, "Diplomatischer Alltag im Spätmittelalter», p. 152) n'avaient habituellement que les lettres de créance et pas de procuration en bonne et due forme, la lettre de créance indiquant l'objet dont ils devaient négocier. La partie anglaise avec laquelle ils négociaient acceptait cela aussi longtemps qu'il ne s'agissait que de négocier des réparations pour des dommages mais lorsque les sujets devinrent plus juridiques et politiques, les représentants anglais exigèrent le respect de la procédure normale; c'est ainsi qu'au début 1435, une ambassade de la Hanse qui devait négocier avec des ambassadeurs anglais à Bruges ne put ouvrir les négociations car les deux diplomates anglais invoquèrent le fait que les diplomates de la Hanse ne pouvaient pas s'accréditer suffisamment et ils n'acceptèrent pas non plus l'excuse donnée par les deux maires de Dantzig et Hambourg selon laquelle la procédure habituelle était celle des simples lettres de créance et des pouvoirs donnés oralement. 
Lubeck, maître Johann Hertze, et cette fois, les négociateurs hanséates furent très efficaces ${ }^{20}$.

Encore faut-il préciser que ces hommes devaient savoir adapter leur art et leur éloquence à l'auditoire et au moment. En 1366, une ambassade florentine se rend à Avignon, pour convaincre le pape Urbain V de retourner en Italie et pour l'assurer du soutien de Florence dans cette entreprise ${ }^{21}$; l'orateur de l'ambassade florentine était Lapo da Castiglioncho, un professeur de droit canon qui était aussi un excellent latiniste; il emmenait avec lui deux discours qu'il allait prononcer devant des auditoires différents: le premier discours, prononcé devant le pape Urbain et un auditoire réduit de conseillers, fut un discours dépourvu de rhétorique dans lequel il présentait concrètement l'offre de la ville de Florence; le second discours en revanche, qu'il tint peu après devant le Consistoire au grand complet, porta sur le même thème mais était farci et même boursouflé de rhétorique; il citait l'exemple d'Alexandre qui avait été jusqu'en Inde, de Scipion l'Africain qui s'était rendu jusque devant Carthage, ceci pour inciter Urbain à ne pas craindre de traverser les Alpes; ce n'est qu'à la fin de son grand discours qu'il reprenait l'offre concrète qu'il avait formulée dans la première harangue. Plus généralement, dans les négociations que menaient les ambassadeurs, il existait une différence fondamentale entre les séances publiques et officielles au cours desquelles l'ambassadeur devait attacher le plus grand soin à la fois à représenter et mettre en scène la majesté de son maître ainsi qu'à défendre scrupuleusement son honneur et ses droits, et les séances "privées» et secrètes, au cours desquelles l'ambassadeur pouvait mener une véritable négociation que nous qualifierions à présent de "gagnant - gagnant».

Certains discours d'ambassadeurs ont paru aux contemporains tellement brillants qu'ils ont ensuite été recopiés et diffusés pour servir de modèle ${ }^{22}$. Un envoyé du prince devait en tout état de cause savoir exercer une vaste palette de fonctions plus ou moins bien définies; la

20. Th. Behrmann, Herrscher und Hansestädte, p. 260.

2I. Th. Haye, "Die lateinische Sprache als Medium mündlicher Diplomatie»; cf. également Id., Oratio.

22. Le discours que Guillaume Fillastre tint en décembre 1459 en réponse à l'ambassadeur français Richard Olivier de Longueil, évêque de Coutances, venu rappeler au duc de Bourgogne ses devoirs vis-à-vis du roi de France, fut jugé tellement remarquable dans sa manière de rétablir les prérogatives du duc de Bourgogne sans pour autant 
formule par laquelle les rois d'Angleterre au XIVe siècle désignaient les tâches assignées à leurs représentants rendait bien cette indécision: "dantes et concedentes eisdem procuratoribus nostris, negotiorum gestoribus, ac nunciis nostris specialibus ... postestatem generalem ${ }^{23}$.

\section{Qui peut représenter le prince?}

Pendant longtemps, on a considéré que seul un personnage non seulement bénéficiant de la confiance du prince mais aussi très proche de lui par le rang et par le sang, ou bien d'une haute dignité, était capable de le représenter et de l'incarner véritablement. Si l'on examine le profil social des ambassadeurs attestés de l'époque carolingienne aux $\mathrm{XI}^{\mathrm{e}}-\mathrm{XII}{ }^{\mathrm{e}}$ siècles, on constate qu'il s'agit souvent de très hauts personnages, notamment des archevêques et des évêques considérés au moins jusqu'à la Réforme grégorienne comme des collègues du souverain.

Le développement de l'écrit et le recours au droit savant ont cependant conduit à développer des instruments permettant de donner délégation: lettres de créance et surtout pouvoirs. A partir de là, il devenait possible de recruter pour les ambassades des personnages de plus petit état; ces mêmes personnages de rang social modeste pouvaient en revanche être des spécialistes.

Certes l'obligation évoquée plus haut de représenter "dignement» le prince qui vous envoyait, de n'être pas trop éloigné du rang des princes que l'on voulait rencontrer, a constamment obligé à envoyer des ambassades dont certains membres étaient d'un haut niveau social. Envoyer à un autre prince des gens de petit état était clairement un affront. Mais en même temps, il fallait adjoindre aux ambassades des spécialistes capables de mener à bien, comme on l'a vu, les «affaires» du prince.

Les cours princières avaient facilement la possibilité de composer des ambassades où les personnages représentatifs côtoyaient les techniciens.

offenser le roi de France qu'il fut reproduit par la suite à plusieurs reprises, sorti de son contexte de naissance (M. Prietzel, «Reden als Waffen der Diplomatie», p. 85 sqq.)

23. Par exemple Foedera, ed. Th. Rymer, t. VII, p. 95; la formule peut accumuler les qualificatifs: suos veros, certos, legitimos, et indubitatos procuratores, actores, factores, negotiorum suorum gestores, et nuncios speciales, et ambaxiatores (ibid., p. 286); le 5 mai 1381 : nostros ambassatores, ac veros, legitimos, et indubitatos procuratores, negotiorum gestores, commissarios et nuncios speciales... (ibid., p. 299). 
Les villes se trouvaient dans une situation beaucoup plus délicate à cet égard. On constate dans leur cas une tendance à s'adresser au XVe siècle, au moment où une véritable diplomatie des cités-états s'affirme, à la mesure de l'importance politique et du rang qu'elles prennent dans le système des états, à des personnages de haut rang. A Bâle par exemple ${ }^{24}$, la politique extérieure de la ville est confiée dans les années 1410-1430 à un petit nombre d'hommes de haut niveau politique et social: Henmann Offenburg en premier lieu et à côté de lui les maires Burkhart zu Rhein et Hans Ludman von Ratperg ainsi que les membres du conseil Ulrich Imhof, Arnold von Ratperg et Wernher Murnhart. Ce sont eux que l'on retrouve régulièrement dans les ambassades qui sont envoyées par la ville. L'inconvénient du système était cependant que ces personnages de haut rang ne se sentaient que partiellement comme les représentants de la ville qui les employait. Henmann Offenburg (1379-1459) à Bâle était passé du commerce au patriciat; il avait adopté volontairement le genre de vie de la noblesse en même temps qu'il devenait le représentant attitré de Bâle à toutes les diètes; mais il était aussi familiaris du souverain ${ }^{25}$ et effectuait pour lui des missions. Walter von Schwarzenberg pour Francfort, Marquart Brisacher pour Constance et Sebald Pfinzig pour Nuremberg correspondent au même type d'ambassadeurs ${ }^{26}$. L'ambivalence de la situation n'était pas toujours facile à gérer: Sebald Pfinzig et Marquart Brisacher décidèrent finalement de renoncer à leur droit de bourgeoisie et à leurs offices urbains pour se consacrer exclusivement au service du roi; Schwarzenberg et Offenburg en revanche n'abandonnèrent pas leur droit de bourgeoisie mais ils accomplirent le service royal en guise d'activité parallèle ${ }^{27}$. A Bâle on reprocha toutefois à Offenburg de trahir les intérêts de la cité et de privilégier les siens, et il dut se défendre en 1445 contre un procès pour trahison dont on le menaçait en arguant du fait que le Dauphin avait voulu lui aussi (après son père Charles VII) lui donner le titre de chambellan. Dans le document

24. E. Gilomen-Schenkel, Henman Offenburg (1379-1459), ein Basler Diplomat im Dienste der Stadt, des Konzils und des Reichs, p. 77.

25. Sur l'importance de ce titre, cf. M. Kintzinger, Westbindungen im spätmittelalterlichen Europa.

26. Sur les ambassadeurs nurembergeois, cf. à présent F. Fuchs und R. Scharf, «Nürnberger Gesandte am Hof Kaiser Friedrichs III".

27. E. Gilomen-Schenkel, Henman Offenburg (1379-1459), ein Basler Diplomat im Dienste der Stadt, des Konzils und des Reichs, p. 57. 
qu'il rédigea alors ${ }^{28}$, il fit valoir qu'il avait énormément travaillé au service des intérêts de Bâle comme diplomate auprès du roi Sigismond, ceci sans être aucunement payé ou défrayé mais en s'entretenant lui-même; s'il avait pu, occasionnellement, s'occuper aussi de ses propres intérêts, on ne pouvait vraiment pas lui en faire reproche; ce fut de toute façon très rare ${ }^{29}$. Il illustrait ce fait - mais en même temps, l'exemple montre l'ambivalence de sa situation - en rapportant comment, vraisemblablement au printemps 1422 alors qu'il séjournait chez le souverain, celui-ci lui aurait proposé de reprendre en gage la prévôté de Bâle qui était alors engagée à la ville pour la somme - faible - de 1000 florins; évidemment il l'aurait payée plus cher mais le marché aurait été profitable au roi (qui touchait la différence) comme à lui; il avait refusé cette offre avantageuse par pur souci des intérêts de la ville et il était parvenu à empêcher le margrave Rudolf von Hochberg-Rötteln de rafler la prévôté: ses habiles négociations permirent à la ville de ne pas perdre la prévôté, ceci au prix d'une simple augmentation du montant de 700 florins; en même temps, la ville obtenait du roi la garantie que cette mise en gage ne pourrait pas être libérée séparément des autres gages d’Empire possédés par la cité, à savoir la douane sur le Rhin et celle de Kleinkems, le tout pour la somme - importante et que le roi aurait donc du mal à réunir - de 8200 florins ${ }^{30}$.

Les villes n'avaient néanmoins qu'une confiance relative vis-à-vis de ces mandataires plus ou moins fiables. A Florence aux XIV et $\mathrm{XV}^{\mathrm{e}}$ siècles, l'on menace de très graves sanctions ceux qui tireraient un bénéfice personnel de leurs missions à l'étranger: toute demande de bénéfice ou de privilège pour soi ou pour sa famille est sévèrement prohibée et punie d'une amende très lourde pouvant être prélevée jusqu'aux petits-enfants du coupable; on veut ainsi faire échec à toute tentative d'emprise familiale sur la politique extérieure de la cité. Mais les réitérations des interdictions de bénéfices personnels lors des ambassades attestent clairement que les familles dirigeantes mesuraient tout le profit possible à retirer de la fréquentation des dirigeants européens et les risques corollaires

28. Basler Chroniken, V, p. 225 sqq.

29. Ibid., p. 228.

30. E. Gilomen-Schenkel, Henman Offenburg (1379-1459), ein Basler Diplomat im Dienste der Stadt, des Konzils und des Reichs, p. 61. 
d'une conduite partisane de la diplomatie ${ }^{31}$. Le phénomène était général: Andrew Offord, ambassadeur d'Edouard III à Avignon en été 1343 n'obtint pas moins de 5 provisions à des bénéfices pour lui-même ${ }^{32}$. En tout état de cause, il existait une limite à ces pratiques qu'il ne fallait pas franchir: Frédéric III fit confisquer les biens à Cologne de son envoyé en France, Heinrich Geisbusch, pour une prétendue conspiration avec le roi Louis XI! ${ }^{33}$

Compte tenu des exigences assignées à la fonction, trouver ces ambassadeurs pouvait être difficile: à Venise, le Sénat était constamment obligé de lutter contre le fait que les nobili, seuls habilités à être ambassadeurs, cherchaient par tous les moyens à se soustraire à leurs obligations. En 1440 par exemple, on déclarait que le prétexte choisi pour refuser une désignation comme ambassadeur - cette désignation n'aurait pas été faite de manière régulière - ne pouvait être accepté; il semble que beaucoup de nobles vénitiens cherchaient à se dérober à la désignation en ne participant pas à la séance du sénat au cours de laquelle ils allaient être nommés. Durant les décennies qui suivent, le problème des refus d'ambassades continue à revenir constamment. En 1500, le sénat alla jusqu’à menacer les ambassadeurs qui refusaient d'une amende de 500 ducats. En décembre 1500 néanmoins, Zaccaria Contarini réussit à faire annuler son choix comme ambassadeur en Hongrie, mais il dut invoquer de multiples raisons: son épouse était malade et il avait dix enfants; l'élection n'avait pas été faite régulièrement; en outre, il avait déjà accompli six ambassades dont trois hors d'Italie, à savoir deux auprès du roi des Romains et une en France - ces ambassades à côté de celles en Hongrie ou auprès du sultan avaient la réputation d'être particulièrement pénibles; lors de son dernier séjour chez Maximilien, il avait dû loger dans une maison où un pestiféré était mort; il était sûr qu'il allait finir comme son père Francesco mort au cours d'une ambassade à Sienne, ou bien comme son oncle Niccolo décédé en Savoie, ou encore comme son grand-père Zaccaria mort en Espagne! ${ }^{34}$

3I. P. Gilli, «De l'importance d'être hors norme», passim.

32. K. Plöger, England and the Avignon Popes, p. 165.

33. P.-J. Heinig, «Römisch-deutscher Herrscherhof und Reichstag im europäischen Gesandscharftssystem an der Wende vom Mittelalter zur Neuzeit», p. 245.

34. Ch. Lutter, Politische Kommunikation an der Wende vom Mittelalter zur Neuzeit, p. 56. 


\section{Un statut juridique de l'ambassadeur?}

L'ambassadeur se dépouillant de sa personnalité pour prendre celle de son maître ne peut être qu'une fiction juridique dont la portée concrète restait à définir. La question du statut de l'ambassadeur concerne deux aspects, l'un vis-à-vis des puissances étrangères, l'autre vis-à-vis de l'intérieur, c'est-à-dire du mandant.

Vis-à-vis de l'extérieur, la question à résoudre était celle de l'immunité diplomatique, garantie certes par le droit romain mais très difficile à faire entrer dans les faits comme le montrent de nombreux exemples: le chroniqueur Jean de Winterthur raconte comment en 1325-1327 un envoyé pontifical qui apportait à Bâle la nouvelle d'une sentence de la Curie défavorable à la ville fut jeté dans le Rhin du haut d'une tour; comme il cherchait encore à s'enfuir à la nage, il fut poursuivi en bateau et tué ${ }^{35}$; plus spectaculaire encore fut l'affaire de l'enlèvement du représentant d'Edouard III à Avignon en 1343, Niccolo de Fieschi, mais elle pourrait avoir eu des conséquences sur l'affirmation de principe de cette immunité diplomatique ${ }^{36}$. En attendant, il restait utile de disposer de sauf-conduits de différentes sortes ${ }^{37}$; encore ceux-ci n'étaient-ils pas toujours efficaces ${ }^{38}$.

La définition «interne» d'un statut de l'ambassadeur n'était pas moins difficile: on retrouve la difficulté de définir ce qu'est un ambassadeur, sachant qu'il doit incarner la personne de son prince mais sans pouvoir faire oublier qu'il n'en est que le substitut. Seuls les papes y sont parvenus au cours du Moyen Age; c'est dû au fait qu'ils ont donné aux légats un pouvoir juridictionnel différencié, depuis ceux, immenses, conférés au légat a latere jusqu'à ceux du simple nuntius ${ }^{39}$. Dès le début

35. Chronica Iohannis Vitodurani, hrsg. von Friedrich Baethgen, p. 101.

36. K. Plöger, «Die Entführung des Fieschi zu Avignon (1340)».

37. M. Kintzinger, "Cum salvo conductu. Geleit im westeuropäischen Spätmittelater».

38. Parmi de nombreuses affaires que l'on pourrait citer, à titre d'exemple, les représailles exercées le 26 octobre 1375 par Edouard III contre le cardinal de Genève coupable d'avoir rançonné un envoyé d'Edouard III, Simon de Burgh, malgré le saufconduit qu'il lui avait préalablement accordé (Foedera, ed. Th. Rymer, t. VII, p. 93-94).

39. Cf. en dernier lieu W. Maleckzek, "Die päpstlichen Legaten im 14. und 15. Jahrhundert»; cf. également S. Weiss, "Delegierte Herrschaft» et B. Studt, "Anspruch und Wirklichkeit»; sur l'essor des légats pontificaux aux XIe-XII siècles, il faut attendre la parution de la thèse d'habilitation de C. Zey, Die päpstliche 
du XIVe siècle, on dispose du corpus normatif quasi définitif réglant l'activité des légats et établissant une hiérarchie fonctionnelle entre les différents types de légats pontificaux: Le legatus de latere est celui qui avait les pouvoirs les plus vastes si bien qu'il était normal qu'il s'agisse d'un cardinal; son rang se marquait dans l'habit, dans le cérémoniel, dans les moyens financiers et dans l'importance de sa suite. En dessous d'eux, Guillaume Durand distingue deux autres types de légats: les legati nati et les legati constituti. Ces légats ont un pouvoir juridictionnel et judiciaire qui s'impose à la juridiction ordinaire des diocèses dans lesquels ils se trouvent. Rares au $\mathrm{XV}^{\mathrm{e}}$ siècle, ils disparaissent à partir de 1460 environ. En revanche, les nuntii - les nonces -, identifiables depuis Innocent III (appelés au XIVe siècle "messagés » en français), sont ceux qui étaient chargés de missions auprès des souverains et notamment de négociations; inférieurs à un légat en rang et en autorité, ils ne disposaient ni de pouvoirs juridictionnels, ni du droit de conférer des bénéfices; leur titre était nuncius et orator sedis apostolicae; on rencontre parmi eux des archevêques ou des évêques mais aussi des clercs de rang moins élevé, appartenant souvent à la bureaucratie pontificale. Ils sont les représentants du pape dont ils accomplissent les ordres suivant le mandat qu'ils ont reçu. Ils pouvaient cependant recevoir des pouvoirs plus étendus comparables à ceux des légats. Ils finiront au $\mathrm{XV}^{\mathrm{e}}$ siècle par remplacer les légats autres que de latere. Les pouvoirs donnés à ces légats sont étendus à une zone géographiquement déterminée, souvent nommée provincia: un ou plusieurs pays, des diocèses, une province ecclésiastique...

La tradition canonique introduisait donc la notion d'officium pour définir le statut de l'ambassadeur. On sort ainsi de la notion de simple représentation de son maître qui reste fondamentale pour les ambassadeurs des rois et des princes qui ne sont en rien ni des officiers ni des magistrats. Pour eux, l'on constate que les termes employés pour les désigner sont variables et il reste longtemps difficile de déterminer un emploi spécifique des termes en rapport avec les missions confiées aux envoyés (du simple port d'une lettre à l'envoi d'un ambassadeur chargé des pleins pouvoirs). On constate que la désignation des ambassadeurs demeure

Legatenpolitik im 11. und 12. Jahrhundert (1049-1081); cf. d'ores et déjà les différentes études de cet auteur mentionnées dans C. Märtl und C. Zey, «Aus der Frühzeit europäischer Diplomatie? Einleitung», p. 15, n. 11. 
très fluctuante dans les statuts urbains du XIV siècle: nuntii, oratores, procuratores, syndici, missi, voire histriones ${ }^{40}$.

Pour les villes en tout cas, il était important de définir un statut de l'ambassadeur fixant ses droits et surtout ses obligations, c'est-à-dire celles d'être fidèle à la mission qui lui avait été confiée. Et l'on retrouve le problème déjà évoqué plus haut du risque du détournement de l'intérêt collectif vers des intérêts privés.

Cela posait aussi la question de la rémunération des ambassadeurs. La norme classique était l'absence de rémunération mais simplement le défraiement et l'espoir d'une récompense pour l'ambassadeur qui avait bien servi son prince. Ce système du défraiement des ambassadeurs était plus ou moins bien organisé selon les royaumes et les principautés; ce sont les rois anglais qui paraissent avoir mis au point le plus précocement un système efficace: les ambassadeurs anglais recevaient avant leur départ une somme d'argent liquide calculée en fonction à la fois d'une estimation en jours de la durée de leur ambassade et de leur rang social; au retour de l'ambassadeur, on procédait à l'apurement des frais en prenant en compte les dépenses exceptionnelles auxquelles l'ambassadeur avait dû faire face ${ }^{41}$; on retrouve des systèmes similaires en Savoie ${ }^{42}$ ou dans l'Etat bourguignon ${ }^{43}$.

40. P. Gilli, «De l'importance d'être hors norme», p. 414.

4I. A. Reitemeier, Aussenpolitik im Spätmittelalter, p. 373-385.

42. E. Pibiri, «En ambassarie devers le roi des Romains», p. 290-293.

43. Pour la Bourgogne à l'époque de Philippe le Bon, cf. désormais A.-B. Spitzbarth, Ambassades et ambassadeurs de Philippe le Bon. Charles le Téméraire a ensuite (cf. P. Ehm, Burgund und das Reich, p. 285 sqq.) soigneusement réglé la question du paiement de ses envoyés; c'était l'Argentier qui en avait la charge mais pour toute somme de plus de $40 \mathrm{~s}$. qu'il payait à un messager ou un ambassadeur, il devait recevoir un ordre de paiement $\mathrm{du}$ duc lui-même outre celui d'un des commissaires aux finances. L'envoyé devait en échange remettre une quittance; les officiers de finances devaient également vérifier si un envoyé pendant son absence de la cour avait continué à figurer sur les ecroes, registres de gages, pour empêcher un double paiement. Ces gages variaient naturellement en fonction de l'importance des ambassadeurs. Avant leur départ, les ambassadeurs recevaient en général une avance "prest» qui devait également être ordonnée par le duc ou les commissaires aux finances. Les paiements des sommes dues étaient cependant souvent très tardifs. 


\section{Vers la professionnalisation des ambassadeurs?}

Pendant longtemps a prévalu l'idée qu'il fallait attendre les ambassadeurs permanents pour rencontrer des diplomates professionnels. Des études récentes ont apporté sur ces questions des nuances importantes en montrant que l'on assiste à une spécialisation de certains ambassadeurs pour certains pays et pour certaines tâches. La première étude à l'avoir fait apparaître est, à ma connaissance, le travail de Christian de Borchgrave sur la diplomatie de Jean sans Peur ${ }^{44}$; il montre que, sous le gouvernement de Jean sans Peur, 227 personnes ont effectué au moins une fois une mission diplomatique; ces 227 "diplomates» ont effectué 939 déplacements ${ }^{45}$. La répartition des déplacements entre eux est tout à fait inégale puisqu'un très grand nombre d'envoyés n'a effectué qu'un tout petit nombre de déplacements tandis que, réciproquement, à peine plus de $10 \%$ de ces envoyés a eu en charge plus de la moitié des ambassades. Concrètement, cela signifie que 28 personnes accomplirent environ 480 missions sur un total de 939, ce qui veut dire une concentration énorme des tâches dans les mains de quelques personnes. Ces 28 personnes - toutes des conseillers nobles ou des légistes de haut niveau en contact régulier avec le duc lui-même - ne sont donc pas des diplomates de métier mais elles constituent tout de même un groupe spécialisé dans l'accomplissement des tâches diplomatiques. Par ailleurs, ces «diplomates» n'accomplissaient pas n'importe quelle mission mais ils avaient leur domaine de spécialité: ainsi Thierry Gherbode avait participé depuis 1387 aux négociations commerciales avec l'Angleterre et il devint sous Jean sans Peur la figure centrale de ces négociations; en revanche ce même Thierry Gherbode n'est pas spécialisé dans le domaine des relations politiques avec l'Angleterre qui est du ressort de gens comme Pierre de la Viefville, Roland d'Uutkerke ou Raoul le Maire; mais il fait à nouveau partie de ceux qui sont compétents pour les négociations avec la Hollande-Zélande et Liège.

Ces constatations ont été récemment fort bien étayées et précisées par plusieurs études récemment parues ou en cours de parution. Pour la Savoie à l'époque d'Amédée VIII, les travaux d'Eva Pibiri font apparaître

44. Ch. de Borchgrave, «Diplomates et diplomaties sous le duc de Bourgogne Jean sans Peur".

45. Ibid., p. 79 sq. 
également cette concentration en un petit nombre de mains et cette spécialisation. Anne-Brigitte Spitzbarth l'a mise en évidence pour la diplomatie de Philippe le Bon dans sa thèse inédite, et la thèse, qui vient tout juste de paraître, de Stéphane Péquignot sur la couronne d'Aragon à l'époque du roi Jacques II (fin XIII - premier quart du XIV ${ }^{\mathrm{e}}$ siècle) établit que, dès le premier quart du XIVe siècle, il en était déjà ainsi en Aragon.

Cette tendance à la spécialisation et à une forme de professionnalisation des ambassadeurs n'avait pas forcément été voulue. Les statuts urbains italiens ${ }^{46}$ s'efforcent certes d'imposer l'obligation de partir en ambassade pour ceux qui ont été désignés ou élus à cette fin (ils sont menacés d'amende ou de privation de responsabilités civiques s'ils refusent de s'acquitter de la charge dans les délais prévus), mais ils veulent en même temps éviter une spécialisation d'un noyau de citoyens dans la représentation extérieure (impossibilité de partir plus d'une fois par an en ambassade, de participer à la fois à des charges électives locales et à une mission d'ambassade...); toutefois, des accommodements sont possibles et apparaissent dans les statuts plus tardifs; ainsi, à Florence dans les statuts de 1415, malgré le devetum qui interdit à tout citoyen d'effectuer plusieurs missions diplomatiques durant un laps de temps de deux ans, les rubriques consacrées aux ambassades précisent que les prieurs peuvent décider d'envoyer à nouveau un citoyen parti depuis peu, à condition d'avoir l'assentiment d'une majorité des seigneurs; cette disposition n'apparaissait pas dans les statuts de 1322-1325.

Au terme de ce parcours, peut-on répondre à la question posée au début de cet exposé: la diplomatie des ambassadeurs était-elle en mesure de prendre le relais de la diplomatie des rencontres princières? Il est clair que des pas importants avaient été faits dans cette direction mais il est clair aussi qu'il serait erroné de dire que les rois, les princes et les citésétats disposaient désormais d'un corps diplomatique professionnel sur lequel ils auraient pu s'appuyer pour gérer leurs relations.

\author{
Jean-Marie Moeglin \\ Université Paris-Sorbonne \\ Ecole pratique des Hautes Etudes
}

46. P. Gilli, «De l'importance d'être hors norme», p. 418. 


\section{BIBLIOGRAPHIE}

\section{Textes}

Basler Chroniken, hrsg. von der Historischen [après le tome 2] und Antiquarischen Gesellschaft in Basel, Leipzig, 7 tomes, 18721902.

Chronica Iohannis Vitodurani, hrsg. von Friedrich Baethgen, Berlin, 1924 (MGH rer. germ. NS. III).

Foedera, conventiones, litterae et cujuscumque generis acta publica inter reges Angliae et alios quosvis imperatores, reges, pontifices, principes vel communitates, ed. Thomas Rymer, London, A. \& J. Churchill, $1704-1735,20 \mathrm{t}$.

\section{Travaux}

Autrand, Françoise, "Ambassade», in Dictionnaire du Moyen Age, éds Claude Gauvard, Alain de Libera et Michel Zink, Paris, PUF, 2002, p. 48.

Behrmann, Thomas, "Hansische Gesandte an Herrscherhöfen: Beobachtungen zum Zeremoniell", in Zeremoniell und Raum. 4. Symposium der Residenzen-Kommission der Akademie zu Göttingen, hrsg. von Werner Paravicini, Sigmaringen, Thorbecke, 1997, p. 97-111.

—, "Vor Stralsund (1370) und Vordingborg (1425). Gesandschaftsverkehr und Königsverhalten im Verlauf zweier dänisch-hansestädtischer Konflikte", in Der Stralsunder Frieden von 1370. Prosopographische Studien, hrsg. von Nils Jörn, Ralf-Gunnar Werlich und Horst Wernicke, Köln/Böhlau, 1998, p. 287-306. 
-, Herrscher und Hansestädte. Studien zum diplomatischen Verkehr im Spätmittelalter, Hamburg, Kovac, 2004 (Greifswalder Historische Studien 6).

BÉLy, Lucien, La société des princes, XVIe - XVIII e siècle, Paris, Fayard, 1999.

Borchgrave, Christophe de, «Diplomates et diplomaties sous le duc de Bourgogne Jean sans Peur", in A la cour de Bourgogne: le duc, son entourage, son train, éd. Jean-Marie Cauchies, Turnhout, Brepols, 1998, p. 67-83.

Chaplais, Pierre, English Medieval Diplomatical Practice (Part I Documents and Intepretation), London, 1975-1982, 2 vols.

—, English Diplomatic Practice in the Middle Ages, London/New York, Hambledon and London, 2003.

Currin, John M., "„Pro expensis Ambassatorum “: diplomacy and financial management in the reign of Henry VII", English Historical Review, 108 (1993), p. 589-609.

Daumet, Georges, Mémoires sur les relations de la France et de la Castille, Paris, Fontemoing, 1913.

Eнм, Petra, Burgund und das Reich. Spätmittelalterliche Aussenpolitik am Beispiel der Regierung Karls des Kühnen (1425-1477), München, Oldenburg, 2002.

Ernst, Fritz, "Über Gesandschaftswesen und Diplomatie an der Wende vom Mittelalter zur Neuzeit", Archiv für Kulturgeschichte, 33 (1951), p. 64-95.

Felten, Franz J., "Verhandlungen an der Kurie im frühen 14. Jahrhundert. Spielregeln der Kommunikation in konfliktgeladenen Beziehungsnetzen", in "Das kommt mir spanisch vor" - Eigenes und Fremdes in den deutsch-spanischen Beziehungen des späten Mittelalters, hrsg. von Klaus Herbers und Nikolaus Jaspert, Münster/Hamburg/Berlin/Wien/London, LIT, 2004, p. 411-474. Fubini, Ricardo, "Classe dirigente e esercizio dalla diplomazia nella Firenze quattrocentesca", in I Ceti dirigenti nella Toscana del Quattrocento, Firenze, 1987, p. 117-189.

-, Quattrocento fiorentino. Politica diplomazia cultura, Ospedaletto (Pisa), Pacini Editore, 1996.

Fuchs, Franz und Scharf, Rainer «Nürnberger Gesandte am Hof Kaiser Friedrichs III", in Aus der Frühzeit europäischer Diplomatie - Zum geistlichen und weltlichen Gesandschaftswesen vom 12. bis 
zum 15. Jahrhundert, hrsg. von Claudia Zey und Claudia Märtl, Zurich, Chronos, 2008, p. 301-330.

GiLli, Patrick, «De l'importance d'être hors norme: la pratique diplomatique de Giannozzo Manetti d'après son biographe Naldo Naldi", in Prêcher la paix et discipliner la société, éd. Rosa Maria Dessì, Turnhout, Brepols, 2005, p. 413-430.

—, «La fonction d'ambassadeur dans les traités juridiques italiens du $\mathrm{XV}^{\mathrm{e}}$ siècle: l'impossible représentation", in La culture juridique en Italie à la fin du Moyen Age, éds Patrick Gilli et Diego Quaglioni, Rome, Ecole française de Rome, à paraître.

Gilomen-Schenkel, Elsanne, Henman Offenburg (1379-1459), ein Basler Diplomat im Dienste der Stadt, des Konzils und des Reichs, Basel, 1975.

Haye, Thomas, Oratio. Mittelalterliche Redekunst in lateinischer Sprache, Leiden, Brill, 1999 (Mittellateinische Studien und Texte 27).

—, "Die lateinische Sprache als Medium mündlicher Diplomatie», in Gesandschafts- und Botenwesen im spätmittelalterlichen Europa, hrsg. von Rainer C. Schwinges und Klaus Wriedt, Ostfildern, Thorbecke, 2003, p. 29-30.

Heinig, Paul-Joachim, "Römisch-deutscher Herrscherhof und Reichstag im europäischen Gesandscharftssystem an der Wende vom Mittelalter zur Neuzeit", in Gesandschafts- und Botenwesen im spätmittelalterlichen Europa, hrsg. von Rainer C. Schwinges und Klaus Wriedt, Ostfildern, Thorbecke, 2003, p. 225-263.

Höflechner, Walter, Beiträge zur Geschichte der Diplomatie und Gesandschaftswesen unter Maximilian I., 1400-1500, Diss., Graz, 1967.

—, «Die Entwicklung österreichischer Diplomatie im Mittelalter und die Aussenpolitik Maximilians I.", in Diplomatie und Aussenpolitik Österreichs, 11. Beiträge zu ihrer Geschichte, hrsg. von Erich Zöllner, Wien, 1977, p. 28-44 (Schriften des Instituts für Österreichskunde 30).

—, "Anmerkungen zu Diplomatie und Gesandschaftswesen des 15. Jahrhunderts", Mitteilungen des österreichischen Staatsarchiv, 32 (1979), p. 1-22.

HüBNER, Klara, «Nüwe mer us Lamparten. Entstehung, Organisation und Funktionsweise spätmittelalterlicher Botenwesen am Beispiel Berns", in Gesandschafts- und Botenwesen im spätmittelalterlichen 
Europa, hrsg. von Rainer C. Schwinges und Klaus Wriedt, Ostfildern, Thorbecke, 2003, p. 265-286.

-, Im Dienste ihrer Stadt - Boten- und Nachrichtenorganisationen in den schweizerisch-oberdeutschen Städten des späten Mittelalters, Osfildern, Thorbecke, à paraître.

Kintzinger, Martin, Westbindungen im spätmittelalterlichen Europa. Auswärtige Politik zwischen dem Reich, Frankreich, Burgund und England in der Regierungszeit Kaiser Sigmunds, Stuttgart, Thorbecke, 2000.

—, "Cum salvo conductu. Geleit im westeuropäischen Spätmittelater», in in Gesandschafts- und Botenwesen im spätmittelalterlichen Europa, hrsg. von Rainer C. Schwinges und Klaus Wriedt, Ostfildern, Thorbecke, 2003, p. 313-363.

LE BIs, Isabelle, "Pratique de la diplomatie. Un dossier d'ambassadeurs français sous Charles VI (1400-1403)", Annuaire-Bulletin de la Société d'histoire de France, (1985-1986), p. 97-215.

Lutter, Christina, Politische Kommunikation an der Wende vom Mittelalter zur Neuzeit. Die diplomatischen Beziehungen zwischen der Republik Venedig und Maximilian (1495-1508), Wien/ München, Oldenburg Verlag, 1998.

Maleckzek, Werner, "Die päpstlichen Legaten im 14. und 15. Jahrhundert», in Gesandschafts- und Botenwesen im spätmittelalterlichen Europa, hrsg. von Rainer C. Schwinges und Klaus Wriedt, Ostfildern, Thorbecke, 2003, p. 33-86.

Märtl, Claudia und Zey, Claudia, "Aus der Frühzeit europäischer Diplomatie? Einleitung", in Aus der Frühzeit europäischer Diplomatie - Zum geistlichen und weltlichen Gesandschaftswesen vom 12. bis zum 15. Jahrhundert, hrsg. von Claudia Zey und Claudia Märtl, Zurich, Chronos, 2008, p. 9-21.

Mattingly, Garrett, Renaissance Diplomacy, London, Jonathan Cape, 1955.

Maulde la Clavière, René de, La diplomatie au temps de Machiavel, Paris, éditions Ernest Leroux, 1892-1893, 3 vols.

Menzel, Viktor, Deutsches Gesandschaftswesen im Mittelalter, Hannover, Hahn, 1892.

Moнr, Andreas, Das Wissen über die anderen - Zur Darstellung fremder Völker in den fränkischen Quellen der Karolingerzeit, Münster, Waxmann, 2005. 
Monnet, Pierre, Recherches sur l'information, la communication et la représentation extérieure des villes allemandes à la fin du Moyen Age, mémoire d'habilitation, 2002, à paraître.

PÉQuignot, Stéphane, "Les instructions aux ambassadeurs des rois d'Aragon (XII ${ }^{\mathrm{e}}-\mathrm{XV}^{\mathrm{e}}$ siècles). Jalons pour l'histoire de la fabrique d'une parole royale efficace", Cahiers d'études hispaniques médiévales, 31 (2008), p. 17-43.

-, Au nom du roi - Pratique diplomatique et pouvoir durant le règne de Jacques II d'Aragon (1291-1327), Madrid, Casa de Velazquez, 2009.

—, "Les diplomaties occidentales et le mouvement du monde", in Histoire du Monde au $X V^{e}$ siècle, éd. Patrick Boucheron, Paris, Fayard, 2009, p. 709-723.

Petersohn, Jürgen, Ein Diplomat des Quattrocento. Angelo Geraldini (1422-1486), Tübingen, Max Niemeyer, 1985.

-, Kaiserlicher Gesandter und Kurienbischof-Andreas Jamometić am Hof Papst Sixtus' IV. (1478-1481) - Aufschlüsse aus neuen Quellen, Hannover, Hahnsche Buchhandlung, 2004.

Pibiri, Eva, "A la recherche d'une épouse. Ambassades et voyages autour des fiançailles d'Annabelle d'Ecosse et de Louis de Savoie, comte de Genève (1444-1445)", in L'itinérance des seigneurs (XIV'-XVIe siècles). Actes du colloque international de Lausanne et Romainmôtier, 29 novembre-1 ${ }^{\text {er }}$ décembre 2001, éds Agostino Paravicini Bagliani, Eva Pibiri et Denis Reynard, Lausanne, 2003, p. 123-171 (Cahiers lausannois d'histoire médiévale, 34).

—, «En ambassarie devers le roi des Romains. Les émissaires du duc Amédée VIII vers l'empereur Sigismond", in La Suisse occidentale et l'Empire, éds Jean-Daniel Morerod, Denis Tappy, Clémence Thévenaz-Modestin et Françoise Vannotti, Lausanne, 2004, p. 289-309 (Société d'histoire de la Suisse romande).

—, «Etre reçu à cour: l'accueil des ambassadeurs étrangers par les ducs Amédée VIII et Louis de Savoie", in L'affermarsi della corte sabauda. Dinastie, poteri, élites in Piemonte e Savoia fra tardo medioevo e prima età moderna, a cura di Paola Bianchi e Luisa C. Gentile, Turin, Silvio Zamorani editore, 2006, p. 77-92.

—, En voyage pour Monseigneur. Ambassadeurs, officiers et messagers à la cour de Savoie (1391-1465), thèse dactylographiée de l'Université 
de Lausanne (2006), à paraître dans les Mémoires et documents publiés par la Société d'histoire de la Suisse romande.

Plöger, Karsten, «Die Entführung des Fieschi zu Avignon (1340) : zur Entwicklung der diplomatischen Immunität in der Frühphase des Hundertjährigen Krieges", Francia, 30 (2003), p. 73-105.

-, England and the Avignon Popes - The practice of Diplomacy in Late Medieval Europe, Oxford, Legenda, 2005.

Prietzel, Malte, "Reden als Waffen der Diplomatie. Rhetorik, Zeremoniell und Politik in den französisch-burgundischen Verhandlungen 1456-1465", in Aussenpolitisches Handeln im ausgehenden Mittelalter: Akteure und Ziele, hrsg. von Sonja Dünnebeil und Christine Ottner, Wien/Köln/Weimar, Böhlau, 2007, p. 73-96.

Queller, Donald, "Thirteenth-Century Diplomatic Envoys: nuncii and procuratores", Speculum, 35 (1960), p. 196-209.

—, Early venetian Legislation on Ambassadors, Geneva, Droz, 1966.

—, The office of Ambassador in the Middle Ages, Princeton, Princeton University Press, 1967.

Reitemeier, Arnd, Aussenpolitik im Spätmittelalter. Die diplomatischen Beziehungen zwischen dem Reich und England (1377-1422), Paderborn, Ferdinand Schöningh, 1999.

—, «Diplomatischer Alltag im Spätmittelalter: Gesandte in den englischen Beziehungen zu Frankreich und zur Hanse", in Gesandschafts- und Botenwesen im spätmittelalterlichen Europa, hrsg. von Rainer C. Schwinges und Klaus Wriedt, Ostfildern, Thorbecke, 2003, p. 135-167.

SAvy, Pierre, "Les ambassadeurs milanais à la cour de Charles le Téméraire», Annales de Bourgogne, 68 (1996), p. 35-56.

Schwinges, Rainer C. und Wriedt, Klaus (Hrsg.), Gesandschafts- und Botenwesen im spätmittelalterlichen Europa, Ostfildern, Thorbecke, 2003 (Vorträge und Forschungen 60).

Spitzbarth, Anne-Brigitte, Ambassades et ambassadeurs de Philippe le Bon, troisième duc Valois de Bourgogne (1419-1467), thèse dactylographiée de l'Université Lille III, 2007, à paraître.

Studt, Birgit, "Anspruch und Wirklichkeit - Der Wandel von Handlungsspielräumen und Reichweite päpstlicher Diplomatie im 15. Jahrhundert", in Aus der Frühzeit europäischer Diplomatie - Zum geistlichen und weltlichen Gesandschaftswesen vom 12. bis 
zum 15. Jahrhundert, hrsg. von Claudia Zey und Claudia Märtl, Zurich, Chronos, 2008, p. 85-118.

Weiss, Stefan, "Delegierte Herrschaft - Innocenz VI., Kardinal Albornoz und die Eroberung des Kirchenstaates", in Aus der Frühzeit europäischer Diplomatie - Zum geistlichen und weltlichen Gesandschaftswesen vom 12. bis zum 15. Jahrhundert, hrsg. von Claudia Zey und Claudia Märtl, Zurich, Chronos, 2008, p. 67-84.

Wriedt, Klaus, "Das gelehrte Personal in der Verwaltung und Diplomatie der Hansestädte», Hansische Geschichtsblätter, 96 (1978), p. 15-37.

Zey, Claudia, Die päpstliche Legatenpolitik im 11. und 12. Jahrhundert (1049-1081). Zur Integration Europas in die werdende Papstkirche, Habilitationsschrift München, à paraître chez Jan Thorbecke Verlag.

ZeY, Claudia und MärTl, Claudia (Hrsg.), Aus der Frühzeit europäischer Diplomatie - Zum geistlichen und weltlichen Gesandschaftswesen vom 12. bis zum 15. Jahrhundert, , Zurich, Chronos, 2008. 
